\title{
Recombinant Tissue Plasminogen Activator Administration in Patients With Cerebrovascular Accident; A Case Series
}

\author{
Hosein Delavar Kasmaei ${ }^{1}$; Alireza Baratloo ${ }^{2, *}$; Zahra Nasiri ${ }^{1}$; Maryam Soleymani ${ }^{1}$; Maryam \\ Oraee Yazdani ${ }^{3}$ \\ ${ }^{1}$ Department of Neurology, Shohadaye Tajrish Hospital, Shahid Beheshti University of Medical Sciences, Tehran, IR Iran \\ ${ }^{2}$ Department of Emergency Medicine, Shohadaye Tajrish Hospital, Shahid Beheshti University of Medical Sciences, Tehran, IR Iran \\ ${ }^{3}$ Department of Medicine, Shahid Beheshti University of Medical Sciences, Tehran, IR Iran \\ *Corresponding author: Alireza Baratloo, Department of Emergency Medicine, Shohadaye Tajrish Hospital, Shahid Beheshti University of Medical Sciences, Tehran, IR Iran. Tel: +98- \\ 9122884364, Fax:+98-2122721155, E-mail: alirezabaratloo@yahoo.com
}

Received: September 7, 2014; Revised: October 8, 2014; Accepted: October 15, 2014

\begin{abstract}
Background: Cerebrovascular accident (CVA), as a debilitating neurological impairment, is one of the most common causes of death in adults. It is observed that CVA is less well-documented in the developing countries compared to the developed ones and most of the available data are based on case series.

Objectives: The current study aimed to evaluate the effectiveness, feasibility, outcome, and safety of intravenous recombinant tissue plasminogen activator (IV rt-PA) administration in patients with acute ischemic cerebrovascular accident(CVA).

Patients and Methods: All CVA patients referred three hours after onset of symptoms to the emergency department (ED) of Shohadaye Tajrish Hospital, Tehran, Iran, from February 2012 to March 2014 were included. The following data were collected using specific check list: demographic data, chief complaint, signs and symptoms, medical history, risk factors, focused neurologic examination, and the National Institutes of Health CVA Scale (NIHSS). Then, all patients received $0.9 \mathrm{mg} / \mathrm{kg}$ of rt-PA as a bolus intravenous dose and intravenous infusion under close monitoring in the ED. Outcomes were categorized as good (complete reversal of focal neurologic deficit), not bad (partial reversal of focal neurologic deficit), death, and unknown (missed follow up). Data were analyzed by STATA software version 11.

Results: Fifteen patients with the mean age of $56.7 \pm 18.9$ years included in the study(male $66.7 \%$ ). The most common chief complaints were left side hemiplegia (46.67\%), right side hemiplegia (40\%), and decreased level of consciousness (13.33\%), respectively. The most common affected vascular territory was medial cerebral artery (MCA) (86.67\%). There was no significant relationship between age $(\mathrm{P}=0.06)$, gender $(\mathrm{P}=0.08)$, NIHSS score $(\mathrm{P}=0.07)$, location of infarct $(\mathrm{P}>0.99)$ and mean time from admission to beginning the drug administration $(\mathrm{P}$ $=0.55$ ) with the outcomes. The outcome in $26.7 \%$ of the patients was favorable and death (two of four) happened due to intra cranial hemorrhage (ICH) as rt-PA side effects.

Conclusions: It seems that intravenous rt-PA administered three hours after CVA is bemeficial in patients with CVA and by reducing the early evaluation time and brain imaging can overcome the CVA complications. However, any conclusion from this study is limited by the small population under study.
\end{abstract}

Keywords:Cerebrovascular Accident; Tissue Plasminogen Activator; Thrombolytic Therapy

\section{Background}

Cerebrovascular accident (CVA), as a debilitating neurological impairment, is one of the most common causes of death in adults, and annually involves approximately 22 million people worldwide. Based on the epidemiologic data, about $2 \%$ to $4 \%$ of the total health care costs globally belong to CVA and more than $4 \%$ of it is spent in developed countries (1). Ischemic CVA is responsible for about three million deaths (two-thirds of CVA deaths) in the developing countries (2). It is observed that CVA is less well-documented in the developing countries compared to the developed ones and most of the available data are based on case series $(2,3)$. Previous studies showed a $42 \%$ decrease of CVA incidence in high income and more than $100 \%$ increase in low and middle income countries (4). Treatment of acute CVA is one of the most challenging medical health problems and many studies have been conducted in this regard during the last 10 to15 years (5). Standard protocols have been designed for appropriate treatment of acute ischemic CVA with recombinant tissue plasminogen activator (rt-PA). It could be applied for patients admitted during three hours window period, but these structures should be modified to apply in the developing countries like Iran (6).

There are some reasons which limit CVA management and treatment in the developing countries such as prehospital delay, because of ignorance regarding the CVA symptoms and the importance of the time window (golden time), financial constraints, and lack of quality and quantity of CVA care. On the other hand, factors such as racial differences in blood coagulation fibrinolysis factors, and

Copyright (C) 2015, Tehran University of Medical Sciences. This is an open-access article distributed under the terms of the Creative Commons Attribution-NonCommercial 4.0 International License (http://creativecommons.org/licenses/by-nc/4.0/) which permits copy and redistribute the material just in noncommercial usages, provided the original work is properly cited. 
genetic variability could affect the cost and efficacy of thrombolysis therapy among the developing and developed countries (3). Therefore further research is needed to make a right decision regarding the issue.

\section{Objectives}

The current study aimed to evaluate the effectiveness, feasibility, outcome, and safety of the intravenous thrombolysis with rt-PA in Iranian acute ischemic CVA patients referred to the emergency department(ED).

\section{Patients and Methods}

This case series was conducted in the emergency and neurology departments of the Shohadaye Tajrish Hospital, an educational medical center in northern Tehran, Iran, from February 2012 to March 2014. The present study evaluated all the patients admitted with acute ischemic CVA diagnosis confirmed by magnetic resonance imaging (MRI) and treated with rt-PA. Patients' demographic data, chief complaint, and symptoms were retrospectively identified by reviewing case files. Also, the medical history, cardiovascular risk factors, and focused neurologic examination were registered and analyzed regarding the findings which led to the suspicion in CVA and assessing the National Institutes of Health CVA Scale (NIHSS). Furthermore, the affected vascular territories were registered. Locations were extracted based on their radiology reports, then reviewed and prospectively confirmed by a neuroimaging specialist. Data collection was performed by hand-searching method via the proper check list. All of the patients were treated with rt-PA (manufactured by Boehringer Company; Germany) by $0.9 \mathrm{mg} / \mathrm{kg}, 10 \%$ of which was administered as a bolus intravenous dose and the rest was given in the following hour as intravenous infusion under close monitoring in the ED. All of the patients were scheduled to conduct carotid doppler ultrasound and echocardiography the next day. The outcomes were categorized as good (complete reversal of focal neurologic deficit), not bad (partial reversal of focal neurologic deficit), death, and unknown (missed follow up). Data were registered in the database designed for this purpose and analyzed with STATA software version 18. Chi-square, Kruskal-Wallis, and fisher's exact tests were used and P-value $<0.05$ considered as the level of significance.

\section{Results}

During the study period, 15 patients were identified with the mean age of $56.7 \pm 18.9$ years (ranging from 23 to 78 ), predominantly consisted of male gender (66.7\%). Statistical tests did not show any significant relationship between age $($ P-value $=0.06)$ and gender $($ P-value $=0.08)$ with the outcome. The most common chief complaints were left side hemiplegia, right side hemiplegia, and decreased level of consciousness, respectively (Figure 1).

The most common outcomes included dysarthria (46.67\%), aphasia (40\%), and dysphasia (13.33\%). The aver- age time from symptom onset to arrival at the ED was $1.1 \pm$ 0.6 hours (ranging from 0.5 to 2.5 hours). The average time from admission to beginning of drug administration was $0.9 \pm 0.3$ hours (ranging from 0.5 to 1.5 hours) that in most of them (86.7\%) lasted more than one hour. There was no significant statistical relationship between the mentioned intervals and the outcome $(\mathrm{P}=0.55)$. The most common affected vascular territory was medial cerebral artery (MCA) that involved 13 (86.7\%) cases (Figure 2). There was no significant relationship between the location of the infarct and the outcome $(\mathrm{P}>0.99)$. The mean NIHSS of the patients was $11.93 \pm 2.98$ (ranging from 9 to 19 ), but there was not any significant relationship between this score and the outcome $(P$-value $=0.07)$. The outcome in four $(26.7 \%)$ cases was favorable, but death was the final outcome in four other ones, and two patients were missed during the period of the study because of early discharge against the medical advice (Figure 3). It should be mentioned that death in two of the four reported cases was due to intra cranial hemorrhage (ICH) that was related to rt-PA side effects. Methadone overdose and electrolyte imbalance were registered as the causes of death in the other two subjects. While, in one patient with intra cranial hemorrhage, the outcome was favorable.

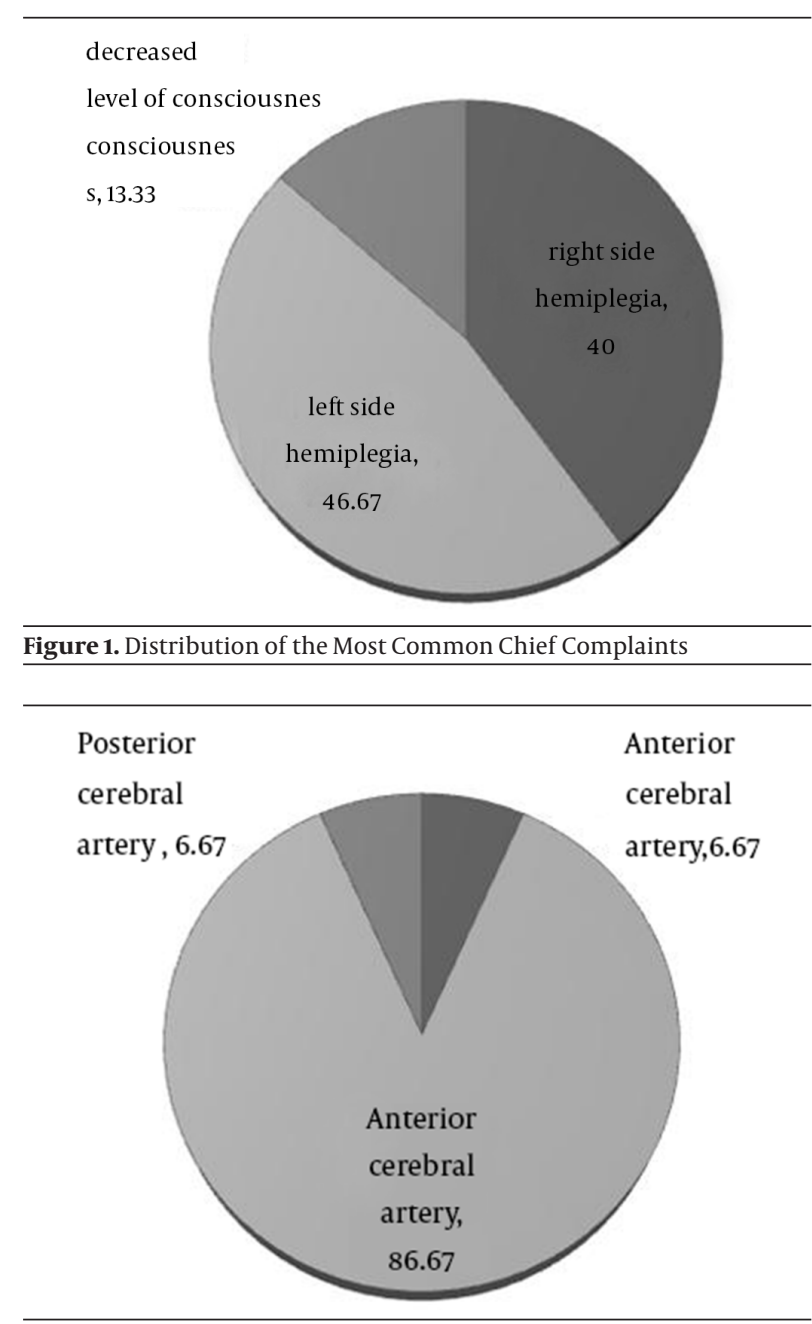


Delavar Kasmaei H et al.

Figure 2. Affected Vascular Territory Distribution

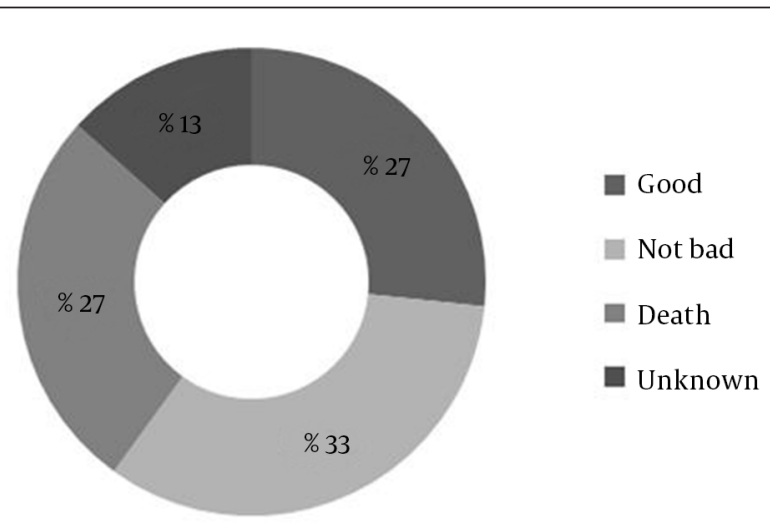

Figure 3. Details of Patients' Outcome Treated With rt-PA

\section{Discussion}

The findings of the present study showed no significant correlation between the studied factors including age, gender, NIHSS scores, location of infract, and the mean time from admission to beginning of drug administration with the patients' outcome. Reports of previous studies indicated that gender may play a role in anatomical location of CVA and CVA subtypes (cardioembolic, atheroembolic and lacunar CVAs), too (7). However, in the current study no significant differences were observed between the anatomical location of the disease in men and women. It should be considered that in women the overall lifetime risk of CVA is higher and the tendency to receive IV thrombolytic therapy is lesser compared to men (7). Therefore, it is recommended to conduct studies based on gender differences to compare the effectiveness of IV thrombolytic therapy. In the present study, CVA was confirmed by Magnetic Resonance Imaging (MRI) in all patients before treatment with rt-PA and the most common site of occlusion (86.67\% of patients) was the medial cerebral artery (MCA), similar to the previous studies. In addition, one case report study showed left MCA territory infarction after using IV rt-PA to treat the right MCA territory ischemic CVA (8). Moreover, another research presented the successful treatment of intra-arterial tissue plasminogen activator for acute ischemic CVA in the left middle cerebral artery (MCA) territory during an early pregnancy (9). Some studies have introduced the MCA as the most common affected site of CVA, which was in line with the findings of the current study. Results of other studies have shown that the tendency for non-contrastenhanced computed tomography application to identify the early signs of ischemic brain infarct or arterial occlusion (hyper dense vessel sign) is increased after administrating IV rt-PA (10). It has also been reported that the use of conventional MRI to detect the artery suscep- tibility signs (magnetic resonance or MR), associated with the hyper dense MCA; is more sensitive than NECT $(11,12)$. MRI can identify both new and old ischemic lesions (11). The potential role of MRI to detect CVA patient with MCA infract was suggested in the earlier studies. It can be helpful to select the best candidates for thrombolytic therapy even more than three hours after CVA onset (11). In agreement with the previous studies, the current study applied MRI to identify and confirm CVA. But, these findings should affect the design of future studies comparing the results of MRI and NECT to distinguish new and preexisting occluded areas, focusing on MCA in Iranian CVA patients. Since the efficacy of thrombolytic therapy on patient's outcome depends on time which leads to the rapid evaluation of CVA, the number of obtained tests should be reduced before management.

Another finding of the current study was death in two of the four reported mortality cases due to intra cranial hemorrhage related to administration of rt-PA. The NINDS CVA Study showed that the risk of symptomatic intracranial hemorrhage, related to administration of rt-PA, increased in patients with high NIHSS score (median NIHSS 20, ranging from 3 to 29). But the favorable outcome was observed even in these high-risk patients compared to those of the placebo and the other patients who had moderately severe CVA (NIHSS score 14) with neurologic improvement (13). Results of the metaanalysis of the 15 published studies using 2639 treated patients were evaluated to compare the treated group of the NINDS rt-PA study. The findings showed that the symptomatic intra-cerebral hemorrhage (ICH) was lower than that of the NINDS study (5.2\% compared to $6.4 \%$ ) with favorable outcome comparable to those of the NINDS trials (14). Lower rate of ICH was observed in the Standard Treatment with Alteplase to Reverse CVA (STARS) study (3.3\% at three days) compared to the rate $(6.4 \%)$ in the NINDS trials (15). In the current study, the mean NIHSS score (11.93 \pm 2.98 , ranging from 9 to 19) was smaller than that of the other study, but no significant relationship was observed between NIHSS score and the outcome $(\mathrm{P}$ value $=0.07$ ). The outcome was favorable only in $26.7 \%$ of the patients, inconsistent with those of the previous studies.

Treatment time is the most important predictor of outcome after CVA. In a review study performed by Mullen et al., the average treatment time with intravenous (IV), intra-arterial (IA), or combined IV+IA thrombolysis was $<6$ hours in different studies (16). In the present study the treatment time in $86.7 \%$ of the patients (ranging from 0.5 to 1.5 hours) lasted more than one hour. In the studied patients a bolus intravenous dose and then an intravenous infusion in the following hour were administered and all of them reached the ED within 0.5 to 2.5 hours after symptom onset. In an updated pooled analysis study of NINDS, ATLANTIS, ECASS II, ECASS III, and EPITHET trials, CVA patients had received $10 \%$ of a total dose ( $0.9 \mathrm{mg} / \mathrm{kg}$ ) of rt-PA (alteplase) as bolus during the first 
minute, then followed by a reminder after one hour. The increase in mortality risk of treatment was observed when the treatment time was longer than 4.5 hours (17). However, in contrast to the results of the current study and the above mentioned studies, the recanalization rate of rt-PA in IA thrombolysis was higher than that of IV rt-PA (up to $80 \%$ ). The difficulty of treatment can be reduced by combining IV and IA therapy and merging the rapid treatment initiation with IV rt-PA followed by IA (16). The current research was performed based on the recommended dose of $0.9 \mathrm{mg} / \mathrm{kg}$ of IV rt-PA used as a drug for thrombolysis therapy of CVA patients in the previous studies, including NINDS, ATLANTIS and ECASS III trials. A study in Japan suggested that the dose of 0.6 $\mathrm{mg} / \mathrm{kg}$ of IV rt-PA could be also beneficial for the treatment of patients within three hours of symptoms onset (18).

Nevertheless, there was a lack of clinical studies which used lower doses of IV rt-PA in the Asian population and a large variation is reported in this regard. (18). Thus, the current study evaluated the outcome in patients using the standard-dose of rt-PA. Considering the findings it can be suggested that further studies evaluate the outcome of lower dose of IV rt-PA in Iranian patients with CVA to reduce the high costs of treatment.

\subsection{Limitations}

There were some limitations in this study. P-value was calculated in a non-significant range, but closely significant which was probably related to the limited number of patients. The low number of treated patients during the time of study may explain these reasons : 1) although different imaging studies, including computed tomography (CT), MRI, computed tomography angiography (CTA), and magnetic resonance angiography are helpful to evaluate CVA, their application is not mentioned in the guideline; because they are time consuming and lead to delay in rt-PA administration;2) Lack of knowledge among people regarding the highest risk of CVA and delay in patients' arrival time;3) Failure to rapid and timely diagnosis by physician, and 4 ) Economic sanctions, that remains as one the important causes of difficulties in rt-PA accessibility. These factors are accounted as limitations of using intravenous rt-PA in CVA treatment in Iran and some other developing countries.

The findings of the present study showed no significant relationship between the studied factors including age, gender, NIHSS scores, location of infract, and the average time from admission to beginning of drug administration with the patients' outcome.

\section{Acknowledgements}

Authors would like to express their special thanks to the staff of Emergency and Neurology Department. This study was a part of the academic thesis of Dr. Maryam
Oraee Yazdani in Shahid Beheshti University of Medical Sciences.

\section{Authors' Contributions}

All the authors have contributed to study the concept and design as well as data interpretation, drafting/revising it for important intellectual content, final approval of the manuscript, and responsible for all aspects of the project.

\section{References}

1. Donnan GA, Fisher M, Macleod M, Davis SM. Stroke. Lancet 2008;371(9624):1612-23.

2. Durai Pandian J,Padma V,Vijaya P, Sylaja PN, Murthy JM. Stroke and thrombolysis in developing countries. Int J Stroke. 2007;2(1):17-26.

3. Ghandehari K. Barriers of thrombolysis therapy in developing countries. Stroke Res Treat. 2011;2011:686797.

4. Feigin VL, Lawes CM, Bennett DA, Barker-Collo SL, Parag V. Worldwide stroke incidence and early case fatality reported in 56 population-based studies: a systematic review. Lancet Neurol. 2009;8(4):355-69.

5. Tosta ED, Rebello LC, Almeida SS, Neiva MS. Treatment of ischemic stroke with r-tPA: implementation challenges in a tertiary hospital in Brazil. Arq Neuropsiquiatr. 2014;72(5):368-72.

6. Ranklev E, Fletcher R. Anaesthetic management of the malignant hyperthermia susceptible parturient. Can J Anaesth. 1987;34(1):106

7. Kent DM, Price LL, Ringleb P, Hill MD, Selker HP. Sex-based differences in response to recombinant tissue plasminogen activator in acute ischemic stroke: a pooled analysis of randomized clinical trials. Stroke. 2005;36(1):62-5.

8. Lai CC, Hu CJ. A left MCA territory infarction during intravenous recombinant tissue plasminogen activator therapy for right MCA territory ischaemic stroke. Emerg Med J. 2006;23(2).

9. Li Y, Margraf J, Kluck B, Jenny D, Castaldo J. Thrombolytic therapy for ischemic stroke secondary to paradoxical embolism in pregnancy: a case report and literature review. Neurologist. 2012;18(1):44-8.

10. Jauch EC, Saver JL, Adams HP, Jr., Bruno A, Connors JJ, Demaerschalk BM, et al. Guidelines for the early management of patients with acute ischemic stroke: a guideline for healthcare professionals from the American Heart Association/American Stroke Association. Stroke. 2013;44(3):870-947.

11. Albers GW. Expanding the window for thrombolytic therapy in acute stroke. The potential role of acute MRI for patient selection. Stroke.1999;30(10):2230-7.

12. Arakawa S, Wright PM, Koga M, Phan TG, Reutens DC, Lim I, et al. Ischemic thresholds for gray and white matter: a diffusion and perfusion magnetic resonance study. Stroke. 2006;37(5):1211-6.

13. Gladstone DJ, Black SE. Update on intravenous tissue plasminogen activator for acute stroke: from clinical trials to clinical practice. CMAJ. 2001;165(3):311-7.

14. Graham GD. Tissue plasminogen activator for acute ischemic stroke in clinical practice: a meta-analysis of safety data. Stroke. 2003;34(12):2847-50.

15. Albers GW, Bates VE, Clark WM, Bell R, Verro P, Hamilton SA. Intravenous tissue-type plasminogen activator for treatment of acute stroke: the Standard Treatment with Alteplase to Reverse Stroke (STARS) study. JAMA. 2000;283(9):1145-50.

16. Mullen MT, Pisapia JM, Tilwa S, Messe SR, Stein SC. Systematic review of outcome after ischemic stroke due to anterior circulation occlusion treated with intravenous, intra-arterial, or combined intravenous+intra-arterial thrombolysis. Stroke. 2012;43(9):23505.

17. Lees KR, Bluhmki E, von Kummer R, Brott TG, Toni D, Grotta JC, et al. Time to treatment with intravenous alteplase and outcome in stroke: an updated pooled analysis of ECASS, ATLANTIS, NINDS, and EPITHET trials. Lancet. 2010;375(9727):1695-703.

18. Ramaiah SS, Yan B. Low-dose tissue plasminogen activator and standard-dose tissue plasminogen activator in acute ischemic stroke in Asian populations: a review. Cerebrovasc Dis 2013;36(3):161-6. 